\title{
Morphological Integration at 50: Patterns and Processes of Integration in Biological Anthropology
}

\author{
Campbell Rolian · Katherine E. Willmore
}

Published online: 26 February 2009

(C) Springer Science+Business Media, LLC 2009

The articles published in this issue of Evolutionary Biology were originally presented in a symposium entitled "Patterns and Processes of Morphological Integration in Primate and Human Evolution", held at the 77th annual meeting of the American Association of Physical Anthropologists in Columbus, Ohio, in April 2008. The symposium marked the 50th anniversary of Everett Olson and Robert Miller's seminal book "Morphological Integration", and sought to capture the current state of affairs in studies of morphological integration, particularly in relation to primate and human evolutionary biology.

In its simplest form, morphological integration is the inter-dependence between sets of traits within an individual, reflecting a common influence from functional and/or developmental factors. The concept of integration is not a new one. In fact, the Principle of the Correlation of Parts, the idea that in order to be viable, organisms must be comprised of organs that are functionally coordinated with each other, was a major theme in the work of French anatomist Georges Cuvier (1769-1832, see Mayr 1982:460). Perhaps not surprisingly, Charles Darwin also discussed this ubiquitous feature of complex organisms in the Origin of Species under the term "Correlation of Growth":

"I mean by this expression that the whole organization is so tied together during its growth and development, that

\section{Rolian $(\bowtie)$}

Department of Cell Biology and Anatomy, University of Calgary, G503-3330 Hospital Dr NW, Calgary, AB T2N 4N1, USA

e-mail: cprolian@ucalgary.ca

\section{K. E. Willmore}

Department of Anthropology, Pennsylvania State University, 409 Carpenter Building, University Park 16802, PA, USA

e-mail: kew20@psu.edu when slight variations in one part occur, and are accumulated through natural selection, other parts become modified" (On the Origin of Species, 1859: 147).

Thus, whereas Cuvier's work emphasized the influence of shared functions on covariation and correlation among traits, Darwin recognized that interactions between these structures during ontogeny could also bias the production of variation within and among traits, and understood the consequences of such a bias on the evolution of complex organisms. Olson and Miller synthesized these earlier views, suggesting that both developmental and functional interactions were important sources of correlation among parts, each contributing towards building 'integrated' organisms in which different parts function in harmony with all others. The process by which such holistic phenotypes are built is what Olson and Miller called morphological integration, i.e., "the summation of the totality of characters which, in their interdependency of form, produce an organism" (1958, p. v). Olson and Miller were also aware of the importance of integration-as a variational property of populations-for the capacity of organismal traits to evolve independently of one another. Olson and Miller also deserve credit for providing the first quantitative methods, mostly based on statistical correlation, for empirically identifying groups of phenotypic traits that are more strongly integrated on the basis of shared developmental and/or functional factors ( $\rho$-groups).

Despite this important insight into a fundamental emergent property of organisms, Olson and Miller's theory of integration did not immediately gain traction among evolutionary biologists. Reasons for this might be that their approach was pattern-based, relying heavily on statistical rather than biological criteria for grouping sets of correlated traits, and inferring in a post-hoc manner developmental and functional reasons for increased correlation among 
traits. In the 1980s, however, morphological integration received renewed attention among evolutionary biologists, but especially physical anthropologists, thanks to a series of studies by James Cheverud on integration in the monkey cranium (e.g., 1982, 1995, 1996). Cheverud placed the study of integration within the theoretical framework of quantitative genetics. Specifically, he applied Lande's work on the evolution of genetic covariance structure to show how organismal covariance structure is patterned according to developmental and functional relationships among traits, ultimately affecting how these traits evolve. Cheverud's approach has had important and lasting methodological and theoretical implications for the study of integration.

On a methodological level, Cheverud's use of the Lande framework established variance/covariance and correlation matrices as a tool of choice for studying interrelationships among traits. This new form of analysis paved the way for the development of matrix-based methods for quantifying and comparing patterns and magnitudes of integration in multivariate datasets (e.g., analysis of eigenvalues, common principal component and factor analyses, matrix correlations). Judging by the methods used in the vast majority of articles in this issue, and the fact that several articles explore new ways of capturing patterns of (co)variation embodied in covariance and correlation matrices (see, e.g., Pavlicev et al., Marroig et al., this volume), matrix-based tools will likely remain popular among students of integration, particularly as the increasing ease and speed of computation afforded by modern PCs enables more complex matrix operations.

At the same time, the development of matrix-based tools facilitated the shift from exploratory to hypothesis-driven research, where for example covariance/correlation matrices derived from multivariate datasets could be tested against theoretical matrices that describe hypothesized relationships among traits determined by functional and/or developmental factors. Using these methods, Cheverud and many others since have confirmed empirically what Cuvier, Darwin and Olson and Miller understood on a more intuitive level: inter-relationships among traits are patterned according to functional and developmental factors, and these patterns not only vary across the different parts of an organism, but also among taxa.

On a theoretical level, these empirical observations based on Lande's framework lead to a refining of integration theory, outlining in particular the mechanisms by which integration itself can evolve. The main ideas behind this expanded theory were first discussed at a 1995 symposium on developmental integration held at the annual meeting of the American Society of Zoologists (now the Society for Integrative and Comparative Biology). In his contribution to that symposium, Cheverud identified four kinds of integration organized hierarchically between individuals and populations. Within individuals, integration is hypothesized to be driven largely by functional and developmental factors, as originally recognized by Olson and Miller. Cheverud stressed, however, that these two forms of integration are not mutually exclusive, because "development can be viewed as dynamic function, and functional integration in the adult is likely to be achieved through developmental integration" (1996: 45).

At the level of populations, morphological traits become genetically integrated when sets of phenotypic traits are inherited together, more or less independently of other sets of traits. Finally, Cheverud proposed that genetic integration leads to evolutionary integration, when morphological traits co-evolve because of a coordinated response to selection. Importantly, Cheverud and others have argued that pleiotropy is a key mechanism linking and driving these four types of integration. For example, at the 1995 symposium, Gunter Wagner suggested that the evolution of traits into integrated sets that become more or less evolutionarily independent of other such sets could occur through the differential suppression of pleiotropic effects between the sets, and the augmentation of these effects among the traits within a set. This revised theory of morphological integration ushered in a new wave of research focusing on process rather than pattern, and stressed the importance of genes and development in structuring covariance among phenotypic traits.

The articles in this issue of Evolutionary Biology illustrate some of these historical changes since 1958, and offer a snapshot of the current diversity of research questions being addressed in studies of morphological integration. With regards to Olson and Miller's original publication, the fundamental concept of integration remains the same, as does the basic methodological approach of looking for increased correlation or covariation among traits. Beyond these similarities, however, there are also important differences. One of these is a relative shift from simply quantifying patterns and magnitudes of integration, to gaining a deeper understanding of the genetic, developmental and functional processes that contribute to these patterns and to their variation across taxa and across organismal structures. Our understanding of the developmental and genetic underpinnings of organismal form have increased exponentially over the past quarter century, enabling us to formulate increasingly refined a priori hypotheses and models for how pleiotropic effects (as measured from a quantitative genetics perspective) and shared developmental factors (e.g., common embryonic origins) affect covariation among a variety of structures (see articles by Bastir and Rosas, Hlusko and Mahaney, MartinezAbadias et al., Willmore et al., Zelditch et al., this volume).

The tendency towards models that test how specific genes or developmental processes affect integration may at first seem like a shift towards a more reductionist and atomistic view of the organism, a shift which is at odds with 
the holistic view of the integrated organism advocated by Olson and Miller. These views are not mutually exclusive, however, and what has emerged from the reductionist approach is the notion that integration is hierarchically structured within organisms. That is, the traits of an organism can display several patterns of integration depending on the organismal level of study (whole organism, specific tissues, cellular) and the hypothesized source of integration (e.g., developmental and/or functional). These patterns of integration can be nested or overlapping, forming subsets of traits that are more or less integrated with each other. In essence, these types of integration studies reveal the modular nature of complex phenotypes. Olson and Miller noted these statistical associations, but considered them to hamper rather than to refine biological interpretations, and actually devised methods to eliminate overlap between sets. Today, modularity is emerging as a fundamental concept in evolutionary biology, and while the concepts of integration and modularity are often juxtaposed, the theoretical and methodological core is the same for each. Both concepts are based on the interdependence between different structures based on developmental, genetic and/or functional factors, and both are quantified by the degree of correlation or covariation among traits. In the context of integration, a module is simply a suite of characters that are more tightly integrated with one another than they are with other characters, and develop, function and evolve largely independently of other such modules (for illustrations of the relationship between integration and modularity, see articles by Bastir and Rosas, Willmore et al., Zelditch et al., this volume).

At face value, these types of studies also suggest that genetic and developmental phenomena have come to play a dominant role in current hypotheses regarding the mechanisms that affect integration and modularity. However, based on the discussion that followed the presentations at the AAPA symposium, and several of the articles in this issue, it is clear that there is a renewed interest in how functional factors influence integration (see Rolian, Young et al., Zelditch et al., this volume). Functionally induced integration has always been harder to define, predict and observe than developmentally based integration. Functional integration occurs when two or more traits participate in a common function and the covariation among these traits affects their joint performance of that function. In contrast to developmental and genetic hypotheses, functional hypotheses require not only information on phenotypic covariation between the traits, but ideally, additional data on how performance is affected in relation to differences in the magnitude and direction of covariation between the traits. In practice, such a research agenda is not realistic, especially if the large samples that are typically required to obtain reliable variance/covariance data have to be matched by an equally large sample looking at functional performance variation in relation to trait covariation.

Furthermore, even with sufficient data on performance variation, we still cannot show conclusively that this functional integration operates independently of genetic and developmental integration. Trying to untangle the specific and independent influences of functional, developmental and genetic factors is an extremely difficult and potentially misleading task. The inextricability of development from function has been emphasized by Cheverud, and researchers are discovering that purely developmental or functional hypotheses generally do not account for all of the patterns observed. In response to this complex and reciprocal relationship between function and development, the focus of several studies has turned towards how epigenetic factors during growth and development, including functions such as mastication (e.g., Zelditch et al., this volume) and locomotion (e.g., Young et al., this volume), interact with the developmental and genetic architecture of an organism to produce patterns of integration. As we gain a better understanding of how specific genetic, developmental and functional factors influence integration, we will likely see more studies combining this information to determine how interactions among different processes during growth and development culminate in observable patterns of integration in the adult phenotype.

Another major shift in morphological integration research illustrated by the symposium has been an increased focus on the evolutionary implications of integration and modularity. Evolutionary significance has always been at the root of integration studies, but with a quantitative genetics framework, and new tools for studying the effects of multiple (albeit simulated) selective regimes, we are now able to explicitly test for the effects of integration and modularity on evolvability, i.e., the ability of the phenotype to respond to selective challenges that can affect the whole organism or only part of it. The connection between integration and modularity on one hand, and the rate and direction of evolutionary change on the other, has been an important facet of Cheverud and Wagner's work on integration. Cheverud's concept of evolutionary integration describes how traits that are inherited together will respond similarly to selective forces, and thus, evolve together. Wagner simultaneously proposed the idea of parcellation, an increasing genetic and phenotypic dissociation of traits, which allow more freedom to vary independently in response to selection (see Wagner 1996, Wagner and Altenberg 1996).

When many traits are more strongly integrated, there is an increased probability that a positive change in one character will incur phenotypic changes in all other characters that are correlated with it. Unless all associated changes are positive or neutral with respect to fitness, the 
original trait that was under positive selection may be constrained in its ability to respond to selection, and thus the possibility for evolutionary change will be limited. If however, an organism is comprised of numerous, smaller modules of correlated traits, or is parcellated, chances increase that a positive change in one of the characters within a module will have a similar effect on the other traits in the module, allowing that suite of structures to change collectively but leaving traits from other modules relatively unaffected. Increased modularity, or parcellation, is thus linked with greater freedom for evolutionary change. This association between patterns of integration/ parcellation and evolvability is empirically evaluated and discussed in several articles in this volume (see Bastir and Rosas, Marroig et al., Martínez-Abadías et al., Porto et al., Rolian). Interestingly, the same patterns of integration and modularity may also be relevant for reconstructing past evolutionary patterns, as illustrated by Ackermann's caution regarding the reconstruction of hominin fossil diversity using different extant models of phenotypic covariation.

As studies embrace the multifaceted nature of integration, and the role that integration plays in evolutionary change, it will be necessary to continue to develop new methods of analysis that account for the complex interactions that we have only touched upon here. Although some new analyses may be modifications of existing methods (see Pavlicev et al., this volume), others may involve a totally new method of quantifying the association among traits. An important oversight of many, if not most, current studies of integration that came up following the symposium, is the lack of interpretation of results that do not fit hypothesized relationships among traits. That is, the results of most studies reveal patterns of integration that cannot readily be explained by the factors initially hypothesized. Generally, researchers casually mention and speculate on these 'deviant' results but do not explicitly pursue new hypotheses to explain them. It is likely that these results are due to the complex interactions among genetic, functional and developmental factors mentioned above, and new or refined methods of analysis will be necessary to uncover the true nature of these interactions.

The symposium and articles in this issue of Evolutionary Biology show that studies of integration over the past 50 years have contributed significantly to our understanding of primate and human evolution. Yet while the symposium focused on the role of integration studies in our understanding of primate and human evolution, the methodological approaches, results and discussions presented at the symposium and in this issue are equally relevant to biologists outside of evolutionary anthropology. As researchers continue to pursue deeper questions and incorporate new tools of analysis, the study of integration will continue to be fruitful for evolutionary biology for years to come.

\section{References}

Cheverud, J. M. (1982). Phenotypic, genetic, and environmental morphological integration in the cranium. Evolution, 36, 499516.

Cheverud, J. M. (1995). Morphological integration in the saddle-back tamarin (Saguinus fuscicollis) cranium. American Naturalist, $145,63-89$.

Cheverud, J. M. (1996). Developmental integration and the evolution of pleiotropy. American Zoologist, 36, 44-50.

Darwin, C. (1859). On the origin of species by means of natural selection. London: J. Murray.

Mayr, E. (1982). The growth of biological thought: diversity, evolution, and inheritance. Cambridge MA: Harvard University Press.

Olson, E. C., \& Miller, R. L. (1958). Morphological integration. Chicago: University of Chicago Press.

Wagner, G. P. (1996). Homologues, natural kinds and the evolution of modularity. American Zoologist, 36, 36-43.

Wagner, G. P., \& Altenberg, L. (1996). Complex adaptations and the evolution of evolvability. Evolution, 50, 967-976. 